\title{
M42 aminopeptidase catalytic site: the structural and functional role of a strictly conserved aspartate residue
}

\author{
Raphael Dutoit ${ }^{1}$, Nathalie Brandt ${ }^{2}$, Tom Van Gompel ${ }^{3}$, Dany Van Elder ${ }^{4}$, Jeroen Van \\ Dyck $^{3}$, Frank Sobott ${ }^{5}$, and Louis Droogmans ${ }^{4}$ \\ ${ }^{1}$ Université Libre de Bruxelles \\ ${ }^{2}$ ULB LABIRIS \\ ${ }^{3}$ Universiteit Antwerpen \\ ${ }^{4} \mathrm{ULB}$ \\ ${ }^{5}$ University of Leeds
}

April 28, 2020

\begin{abstract}
The M42 aminopeptidases are a family of dinuclear aminopeptidases widely distributed in Prokaryotes. They are potentially associated to the proteasome, achieving complete peptide destruction. Their most peculiar characteristic is their quaternary structure, a tetrahedron-shaped particle made of twelve subunits. The catalytic site of M42 aminopeptidases is defined by seven conserved residues. Five of them are involved in metal ion binding which is important to maintain both the activity and the oligomeric state. The sixth conserved residue, a glutamate, is the catalytic base deprotonating the water molecule during peptide bond hydrolysis. The seventh residue is an aspartate whose function remains poorly understood. This aspartate residue, however, must have a critical role as it is strictly conserved in all MH clan enzymes. It forms some kind of catalytic triad with the histidine residue and the metal ion of the M2 binding site. We assess its role in TmPep1050, an M42 aminopeptidase of Thermotoga maritima, through a mutational approach. Asp-62 was substituted with alanine, asparagine, or glutamate residue. The three Asp-62 substitutions completely abolished TmPep1050 activity and impeded dodecamer formation. They also interfered with metal ion binding as only one cobalt ion is bound per subunit instead of two. The structural data showed that the Asp62Ala substitution has an impact on the active site folds becoming similar to TmPep1050 dimer. We propose a structural role for Asp-62, helping to stabilize a crucial loop in the active site and to position correctly the catalytic base and a metal ion ligand of the M1 site.
\end{abstract}

\section{Hosted file}

manuscript_proteins_rdutoit.pdf available at https://authorea.com/users/307628/articles/438634m42-aminopeptidase-catalytic-site-the-structural-and-functional-role-of-a-strictly-conservedaspartate-residue 

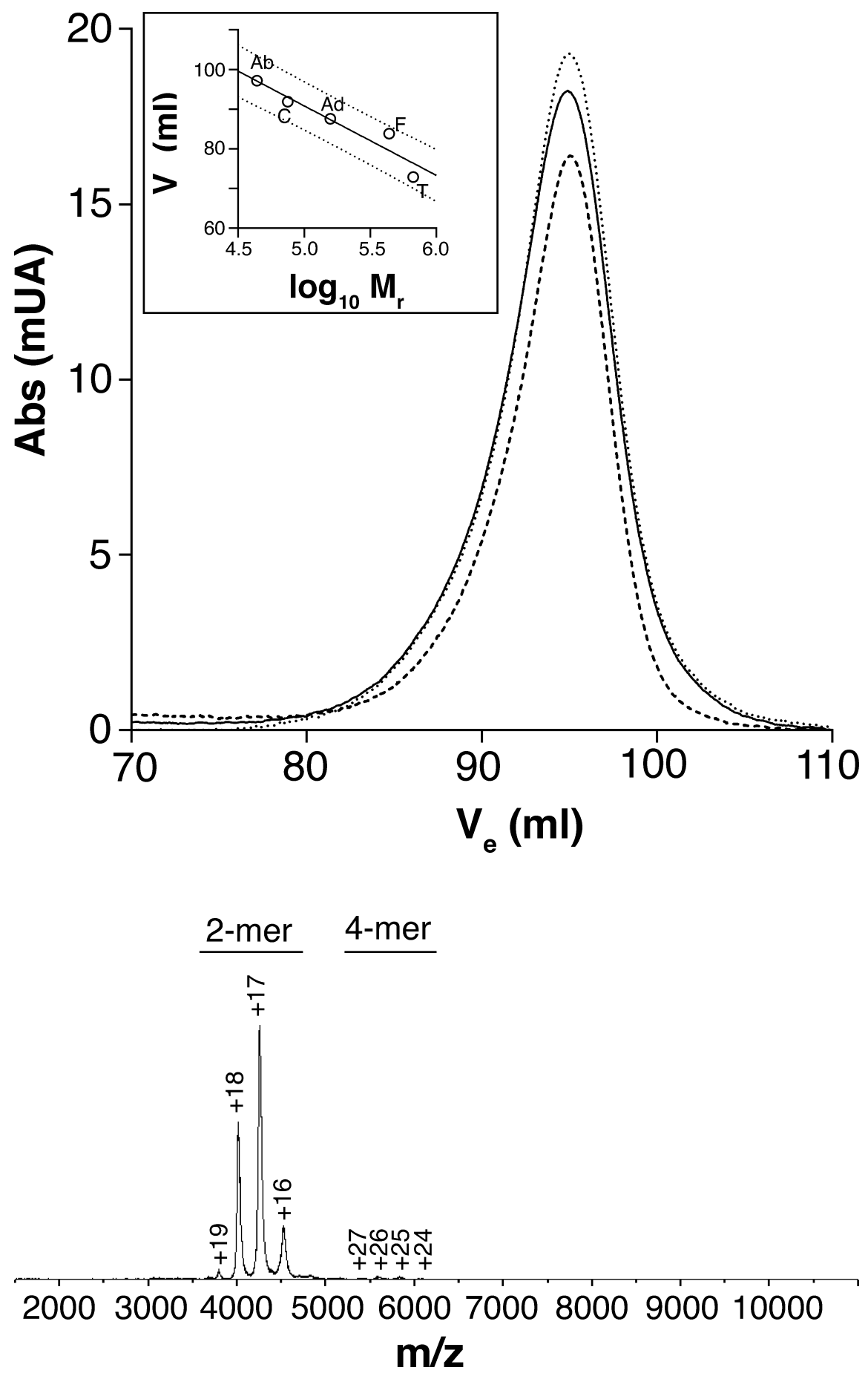

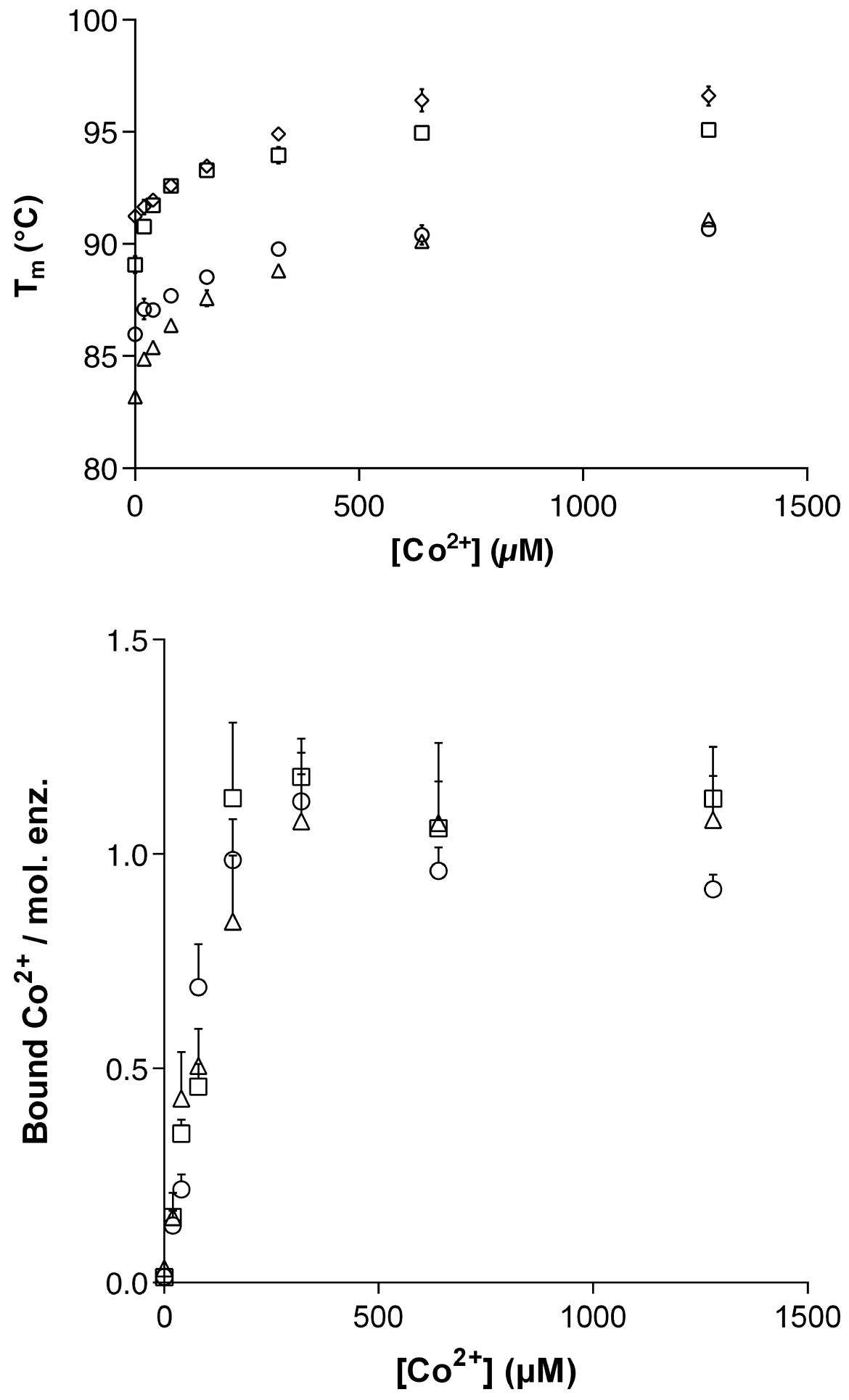


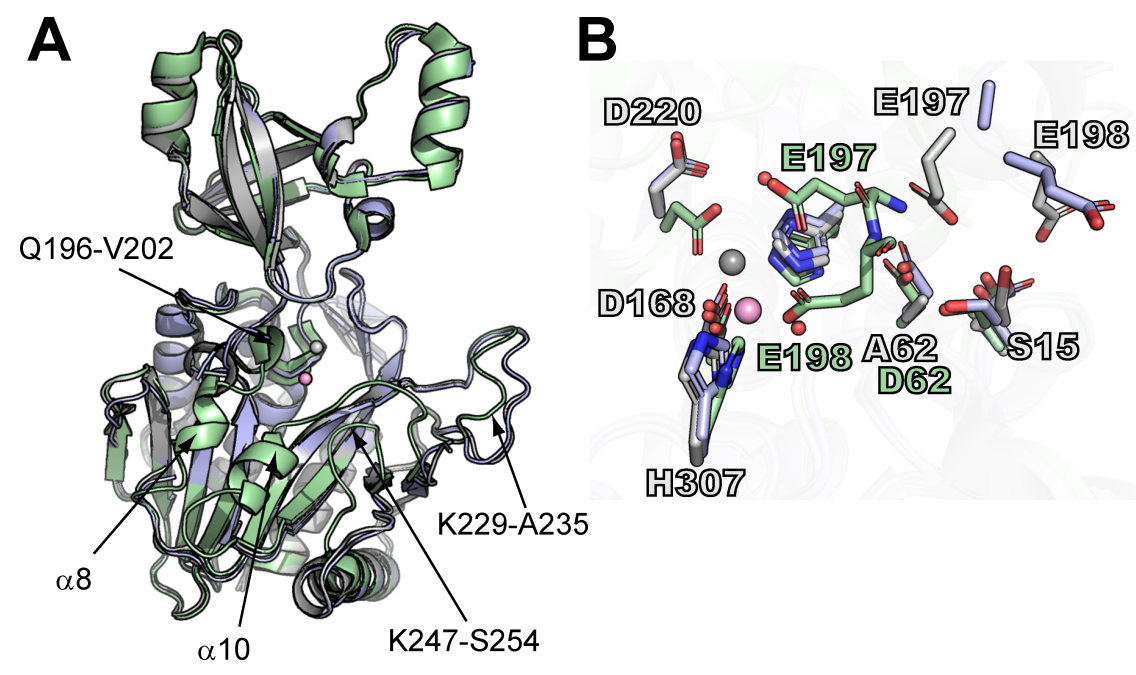

